\title{
Simplex Equations and Their Solutions
}

\author{
Igor Frenkel $^{1}$ and Gregory Moore ${ }^{2 \star}$ \\ 1 Department of Mathematics, Yale University, New Haven, CT 06520, USA \\ 2 Department of Physics, Yale University, New Haven, CT 06511, USA
}

Received August 16, 1990

\begin{abstract}
We investigate an $n$-simplex generalization of the classical and quantum Yang-Baxter equation. For the case of $\operatorname{sl}(2)$ we find the most general solution of the classical $n$-simplex equation for all $n$. These classical solutions can be quantized (in the sense of quantum group theory) for $n=2,3$ and we exhibit a quantum solution to the tetrahedron equations $(n=3)$. The classical nondegenerate solutions cannot be quantized for $n=4$.
\end{abstract}

\section{Introduction}

Conformal field theory has recently emerged as a powerful unifying principle for seemingly disparate mathematical theories such as representations of loop groups, the theory of Riemann surfaces and moduli spaces, quantum groups, and three dimensional topology. This new field is far from completion as a rigorous mathematical theory but the general structure and the key ideas are rather clear. From the physical point of view, conformal field theory can be considered as a simple nontrivial example of a quantum field theory. Although quantum field theory has been extensively developed by physicists during the past several decades, the mathematical structure of quantum field theory has been thoroughly understood only in the cases of free field theories and conformal field theories. The latter have already yielded surprising and far-reaching results. It may therefore be expected that other more sophisticated quantum field theories will be related to correspondingly deeper questions in mathematics. One of the motivations of the present paper was the search for mathematical structures in other quantum field theories which have clear analogies to those arising in conformal field theory.

A simple and basic ingredient in conformal field theory (following from the consistency of the operator product expansion) is the triangular, or Yang-Baxter

\footnotetext{
^ (moore@yalphy.hepnet, or @yalehep.bitnet)
} 
equation,

$$
R_{12} R_{13} R_{23}=R_{23} R_{13} R_{12},
$$

where $R \in$ End $(V \otimes V)$ for some vector space $V$ and $R_{12}$ denotes $R \otimes I$, and similarly $R_{13}$ and $R_{23}$ are elements of $\operatorname{End}(V \otimes V \otimes V)$. The triangular equation is directly related to the representations of braid groups and solutions give rise to applications to knot and link invariants as well as invariants of three-dimensional manifolds. The general theory of quantum groups provides a universal construction of a one-parameter solution of the triangular equation associated to any finitedimensional simple Lie algebra together with a representation $V$. The simplest example of the Lie algebra $s l(2)$ together with its two-dimensional representation gives the celebrated solution [1]

$$
R^{(2)}=\left(\begin{array}{cccc}
q & 0 & 0 & 0 \\
0 & 1 & q-q^{-1} & 0 \\
0 & 0 & 1 & 0 \\
0 & 0 & 0 & q
\end{array}\right)
$$

associated with the 6-vertex model of statistical mechanics, the Jones polynomials and, when $q$ is a root of unity, invariants of three-dimensional manifolds $[2,3]$. Moreover, this solution can itself be used as a starting point for defining a quantum group [4-6].

The general form of $R$ for any simple Lie algebra and any representation $V$ is much more involved, and, in order to understand better these complicated formulae it is sometimes useful to take advantage of the existence of the parameter $q$ and examine instead the first term in an expansion in the variable $t=\log q$. The resulting expansion,

$$
R=1+t r+\mathcal{O}\left(t^{2}\right),
$$

defines an operator $r \in \operatorname{End}(V \otimes V)$ which satisfies the "classical triangular equation"

$$
\left[r_{12}, r_{23}\right]+\left[r_{12}, r_{23}\right]+\left[r_{13}, r_{23}\right]=0 \text {, }
$$

where $r_{12}$ corresponds to $R_{12}$, etc. This "semiclassical expansion" is useful in part because it is much easier to describe the universal solution of the classical triangular equation than it is to describe its quantum counterpart. The formula is $r_{12}=\pi \otimes \pi(\tilde{r})$, where $\pi$ is a representation of a Lie algebra $g$ in $V$ and $\tilde{r} \in g \otimes g$ satisfying the analogue of (1.4) in $g \otimes g \otimes g$ is given by the "half-Casimir." For example; for $g=\operatorname{sl}(2)$ one has

$$
\tilde{r}=h \otimes h+4 e \otimes f,
$$

where $e, f, h$ are generators of $\operatorname{sl}(2)$ satisfying $[h, e]=2 e,[h, f]=-2 f,[e, f]=h$. Clearly, for the two-dimensional representation ${ }^{1}$

$$
h=\left(\begin{array}{cc}
1 & 0 \\
0 & -1
\end{array}\right), \quad e=\left(\begin{array}{ll}
0 & 1 \\
0 & 0
\end{array}\right), \quad f=\left(\begin{array}{ll}
0 & 0 \\
1 & 0
\end{array}\right),
$$

\footnotetext{
${ }^{1}$ We will not distinguish notationally between the Lie algebra generators $e, f, h$ and their two-dimensional representation, since the appropriate meaning is clear from the context. Similarly we will drop the tilde from $r$ in (1.5), etc
} 
$\pi \otimes \pi(\tilde{r})$ gives the infinitesimal generator of $R$ in (1.2), since the latter may be written as

$$
R=q^{1 / 2} q^{(1 / 2) h \otimes h}\left(1+\left(q-q^{-1}\right) e \otimes f\right) .
$$

Thus we may refer to $R$ as a quantization of the classical $r$ matrix [4-6].

A natural generalization of the triangular equation is the tetrahedral equation, one version of which was introduced ten years ago by Zamalodchikov while investigating three-dimensional integrable statistical mechanical systems [7]. We define the tetrahedral equation to be the equation

$$
R_{123} R_{124} R_{134} R_{234}=R_{234} R_{134} R_{124} R_{123},
$$

where $R \in \operatorname{End}(V \otimes V \otimes V)$ for some vector space $V$ and $R_{123}$ denotes $R \otimes 1$, etc. as in the triangular equation ${ }^{2}$. One can simplify the notation by introducing $R_{1}=R_{234}$ and so forth. Moreover, introducing

$$
R_{i} \equiv R_{1,2, \ldots, i-1, i+1, \ldots, n+1}
$$

we define the general $n$-simplex equation by the formula:

$$
R_{1} R_{2} \cdots R_{n+1}=R_{n+1} \cdots R_{2} R_{1}
$$

where $R_{i} \in \operatorname{End}\left(V^{\otimes(n+1)}\right)$. The corresponding classical simplex equation is therefore

$$
\sum_{i<j}\left[r_{i}, r_{j}\right]=0,
$$

where $r_{i} \in g^{\otimes(n+1)}$ and the identity (1.11) is understood in $\mathscr{U} g^{\otimes(n+1)}$, where $\mathscr{U} g$ is the universal enveloping algebra of $g$. The investigation of the solutions of Eqs. (1.10) and (1.11) for the case $g=s l(2)$ is the subject of the present paper. Variants of these equations have been previously investigated in [7-11].

One may of course identify many solutions of (1.10) and (1.11) by inspection, since for example we may choose commutative solutions satisfying $\left[R_{i}, R_{j}\right]=0$ or $\left[r_{i}, r_{j}\right]=0$ for all $i, j=1, \ldots, n+1$. On the other hand, one should feel that nontrivial solutions to these equations are unlikely to exist as $n$ increases since for $V$ of dimension $d$ we have $d^{n}(d-1)$ more equations than variables.

Contrary to this natural skepticism, our first result, proved in Sect. 2, states that the classical simplex equation has exactly the same parameter space of solutions as the initial Yang-Baxter equation. The quantum equation, as usual, presents many more difficulties. Our second result, proved in Sect. 3, is the construction of a nontrivial solution of the quantum tetrahedron equation corresponding to the nondegenerate classical solutions. Finally, our third result, proved in Sect. 4 states that the nondegenerate classical solutions for $n=4$ cannot be quantized: There exists no $R$ matrix satisfying the $n$-simplex equation with a nondegenerate classical solution as a classical limit, at least for $n=4$.

We would like to stress that the methods that we are using are completely elementary, although they probably indicate the existence of a new nontrivial algebraic structure. Since the algebraic structure underlying the quantum solution

${ }^{2}$ Equation (1.8) differs from Zamolodchikov's in the structure of the operators. The two equations are compared in Appendix A 
of the triangular equation is known to be a quantum group, the solution of the quantum tetrahedral equation opens the path beyond quantum groups.

Our results suggest many interesting open problems. Some of these are mentioned below. Here we would like to point out that there are possible interesting applications to topology. Just as (1.2) yields invariants of links in three dimensions, it might be that our solution provides invariants of knotted 2-spheres in four dimensions. An analog of the Reidemeister moves for this situation is known and the main nontrivial move (analogous to the Reidemeister move of type III) corresponds to the tetrahedron equation [12]. It is also natural to expect that our solution has connections to invariants of 4-manifolds.

The nonexistence of a quantization of the classical $r$ matrix for $n=4$ is striking, and is in accord with the general point of view espoused in [13-15]. It was shown in [2] that integrable field theories and nontrivial fixed points of the beta function in two-dimensional quantum field theory are connected to topology and gauge theory in three dimensions. One may wonder if this kind of connection will work in higher dimensions. Both quantum field theory and geometry become simpler in higher dimensions: It is not possible to define nontrivially interacting quantum field theories in greater than four dimensions, and even in four dimensions one must introduce nonabelian gauge fields to define a sensible theory. Similarly, the Poincaré conjecture and other aspects of topology and geometry become (relatively) simpler in higher dimensions and hence the dimensions in which geometry and quantum field theory are most interesting coincide. It is thus startling that it is only in the cases relevant to three and four dimensional topology that the purely algebraic $n$-simplex equations also admit nontrivial solutions. According to the above picture, it is natural to expect that the $n=3$ solution will have connections to four-dimensional gauge theory. The nonexistence of a nontrivial $n=4$ solution may be taken as evidence for this general scheme. It thus might be that the purely algebraic $n$-simplex equations manage to capture something of the essential nature of quantum field theory and geometry.

\section{Classical $n$-Simplex Equations}

In this section we find the most general solution of Eq. (1.11) for the case that $r \in \operatorname{sl}(2)^{\otimes n}$. We begin with $n=2$. In this case there are two classes of solutions:

Proposition 1. The most general solution of (1.4) for sl(2) is either

a) $r=v \otimes v$, for some $v \in \operatorname{sl}(2)$, or,

b)

$$
\begin{aligned}
r= & r(a, b, c, d) \\
\equiv & a\left(J_{1} \otimes J_{1}+J_{2} \otimes J_{2}+J_{3} \otimes J_{3}\right)+b\left(J_{1} \otimes J_{2}-J_{2} \otimes J_{1}\right) \\
& +c\left(J_{1} \otimes J_{3}-J_{3} \otimes J_{1}\right)+d\left(J_{2} \otimes J_{3}-J_{3} \otimes J_{2}\right),
\end{aligned}
$$

where $J_{i}$ are a basis for $\operatorname{sl}(2)$ with $\left[J_{i}, J_{j}\right]=\varepsilon_{i j k} J_{k}$ and

$$
a^{2}+b^{2}+c^{2}+d^{2}=0 .
$$

Proof. Direct calculation. 
Using Proposition 1 we can construct the most general possible ansatz for a solution to (1.11) for $n=3$ as follows. If $r$ satisfies (1.11) for $n=3$ then we may decompose it with respect to the first index, i.e., $r=\sum_{i} J_{i} \otimes Q_{i}$. Plugging into (1.11), decomposing with respect to the first factor, and using the identity $[a \otimes b, c \otimes d]=$ $\frac{1}{2}\{a, c\} \otimes[b, d]+\frac{1}{2}[a, c] \otimes\{b, d\}$ we find that a necessary condition for $r$ to solve (1.11) is that $Q_{i}+Q_{j}$ must be solutions of (1.4) for all pairs of indices $i, j$. It follows from Proposition 1 that $Q_{i}=r\left(a_{i}, b_{i}, c_{i}, d_{i}\right)$ for some 4-vectors satisfying (2.2), and moreover that the 4-vectors $\left(a_{i}, b_{i}, c_{i}, d_{i}\right)$ must be mutually orthogonal. Furthermore, one can easily check that solutions to (1.4) of type (a) cannot be added to solutions of (1.4) of type (b) to produce new solutions.

By the same token we may decompose $r$ with respect to the last index according to $r=\sum_{i} V_{i} \otimes J_{i}$. Requiring that $V_{i}$ be of the form $V_{i}=r\left(a_{i}^{\prime}, b_{i}^{\prime}, c_{i}^{\prime}, d_{i}^{\prime}\right)$ forces:

$$
\begin{aligned}
& \left(a_{2}, b_{2}, c_{2}, d_{2}\right)=\left(b_{1},-a_{1},-d_{1}, c_{1}\right), \\
& \left(a_{3}, b_{3}, c_{3}, d_{3}\right)=\left(c_{1}, d_{1},-a_{1},-b_{1}\right),
\end{aligned}
$$

so that the orthogonality constraints are automatically satisfied. One may continue this process to establish

Proposition 2. Any nondegenerate solution to the classical $n$-simplex equation for sl(2) must be of the form

$$
r=S^{n}(A) \equiv \sum_{l_{1}, \ldots, l_{n}} J_{l_{1}} \otimes \cdots \otimes J_{l_{n}} \operatorname{Re}\left[l_{1} \cdots l_{n} A\right]
$$

where the sum runs over the imaginary quaternions $l \in\{i, j, k\}, J_{1}=J_{i}, J_{2}=J_{j}, J_{3}=J_{k}$, and $A=a+b i+c j+d k$ with

$$
a^{2}+b^{2}+c^{2}+d^{2}=0 .
$$

Note that we are working with the quaternions "over the complex numbers," so for a quaternion $B=b_{0} 1+b_{1} i+b_{2} j+b_{3} k$ with $b_{i}$ complex we have $\operatorname{Re}(B) \equiv b_{0}$. Alternatively, we can represent $1, i, j, k$ by two-by-two matrices and replace $\operatorname{Re}$ by half the trace.

It remains to check that the $S^{n}(A)$ are in fact solutions of (1.11). This may be established by an induetive argument which reveals that the $S^{n}(A)$ satisfy several interesting identities:

Theorem 1. We have the identities

1)

2)

$$
\sum_{l<l^{\prime}}\left[S_{l}^{n}(A), S_{l^{\prime}}^{n}(A)\right]=0
$$

$$
\sum_{l=1}^{n}\left[S^{n}(A), S_{l}^{n-1}(A i)\right]+\frac{1}{2} \sum_{1 \leqq l<l^{\prime} \leqq n}\left(\left\{S_{l}^{n-1}(A j), S_{l^{\prime}}^{n-1}(A k)\right\}-\left\{S_{l}^{n-1}(A k), S_{l^{\prime}}^{n-1}(A j)\right\}\right)=0 .
$$

Together with two cyclic permutations on the imaginary quaternions.

3)

$$
\left[S^{n}\left(A^{\prime}\right), S^{n}\left(A^{\prime \prime}\right)\right]+\frac{1}{2} \sum_{l=1}^{n}\left(\left\{S^{n}\left(A^{\prime \prime}\right), S_{l}^{n-1}\left(A^{\prime}\right)\right\}-\left\{S^{n}\left(A^{\prime}\right), S_{l}^{n-1}\left(A^{\prime \prime}\right)\right\}\right)=0
$$


where $A^{\prime}, A^{\prime \prime}$ can be any of $A, A i, A j, A k$,

4)

$$
\sum_{l=1}^{n}\left[S^{n}(A), S_{l}^{n-1}(A)\right]=0 .
$$

Proof. Let us consider first the inductive step. The equations at step $n+1$ may be reduced to those at step $n$ using the identities

$$
\begin{aligned}
& S_{1}^{n}(A)=1 \otimes S^{n}(A) \\
& S_{l}^{n}(A)=J_{1} \otimes S_{l-1}^{n-1}(A i)+J_{2} \otimes S_{l-1}^{n-1}(A j)+J_{3} \otimes S_{l-1}^{n-1}(A k)
\end{aligned}
$$

for $l>1$, together with the elementary equations

$$
\begin{aligned}
& {[a \otimes b, c \otimes d]=\frac{1}{2}\{a, c\} \otimes[b, d]+\frac{1}{2}[a, c] \otimes\{b, d\},} \\
& \{a \otimes b, c \otimes d\}=\frac{1}{2}[a, c] \otimes[b, d]+\frac{1}{2}\{a, c\} \otimes\{b, d\} .
\end{aligned}
$$

For example, decomposing the classical $(n+1)$-simplex equation, identity one above, with respect to the first factor gives the classical $n$-simplex equation multiplying terms of the form $\left\{J_{i}, J_{i}\right\}$. Terms of the form $\left\{J_{i}, J_{j}\right\}$ with $i \neq j$ multiply

$$
\sum_{l<l^{\prime}}\left[S_{l}^{n}(A i), S_{l^{\prime}}^{n}(A j)\right]+\left[S_{l}^{n}(A j), S_{l^{\prime}}^{n}(A i)\right]
$$

Notice that if $A$ satisfies (2.4) then so does $A(i+j)$ and hence $S^{n}(A i)+S^{n}(A j)$ satisfies the $n$ simplex equation by the inductive hypothesis. From this one may deduce that the expression in (2.10) vanishes. Terms multiplying $J_{1}, J_{2}, J_{3}$ in the first factor multiply identities of type 2 .

Continuing as above we may decompose identities of type 2 at step $n+1$ to identities at step $n$. All terms multiplying anticommutators $\{J, J\}$ in the first factor vanish by identities of type 2 at level $n$. Terms multiplying $J_{1}, J_{2}, J_{3}$ in the first factor vanish by the $n$-simplex equation or by identities of type 3 , at level $n$.

Decomposing identity 3 at level $n+1$ gives anticommutator terms multiplying identities of type 3 at level $n$, while terms multiplying $J_{1}, J_{2}, J_{3}$ multiply either identities of type 2 or expressions like:

$$
\begin{aligned}
& -\left(\left(S^{n}(A)\right)^{2}+\left(S^{n}(A i)\right)^{2}+\left(S^{n}(A j)\right)^{2}+\left(S^{n}(A k)\right)^{2}\right) \\
& \quad+\frac{1}{2} \sum_{l}\left[S^{n}(A), S_{l}^{n-1}(A)\right]+\frac{1}{2} \sum\left[S^{n}(A k), S_{l}^{n-1}(A k)\right] .
\end{aligned}
$$

From the (2.4) it follows that, for any quaternions $\alpha, \beta$,

$$
\operatorname{Re}(\alpha A) \operatorname{Re}(\beta A)+\operatorname{Re}(\alpha A i) \operatorname{Re}(\beta A i)+\operatorname{Re}(\alpha A j) \operatorname{Re}(\beta A j)+\operatorname{Re}(\alpha A k) \operatorname{Re}(\beta A k)=0,
$$

and hence the first line in (2.11) sums to zero. The two remaining sums vanish by identity 4.

Decomposing identity 4 we find anticommutator terms multiply identity 2 or identity 4 while terms multiplying $J_{1}, J_{2}, J_{3}$ can be converted to identity 2 using again the identity (2.12).

Finally, one can check directly that the identities are satisfied for $n=2$, thus completing the proof. 


\section{Quantum Tetrahedral Solution}

We now turn to $n=3$. Choosing $A=1+\sqrt{-1} k$ we seek a quantization of

$$
r=h \otimes h \otimes h+4 h \otimes e \otimes f-4 e \otimes h \otimes f+4 e \otimes f \otimes h .
$$

Given the central importance of the solution (1.2) for $n=2$ it is natural to search for a solution in the tensor product of spin 1/2 representations. In this case we have

Theorem 2. The $8 \times 8$ matrix

$$
R^{(3)}=q^{1 / 2} q^{(1 / 2) h \otimes h \otimes h}\left[1+\left(q-q^{-1}\right)\left(h \otimes e \otimes f-e \otimes h q^{-h} \otimes f+e \otimes f \otimes h\right)\right]
$$

is a solution of the quantum tetrahedral equation:

$$
R_{1} R_{2} R_{3} R_{4}=R_{4} R_{3} R_{2} R_{1} \text {. }
$$

Proof. The proof proceeds in several steps, reducing the tetrahedral equation to simpler identities. We begin with some simple reductions. Let $X$ be any operator in $V \otimes V \otimes V \otimes V$, where $V$ is the two-dimensional representation of $\operatorname{sl}(2)$. It is convenient to define

$$
h_{1}=\left(\begin{array}{ll}
1 & 0 \\
0 & 0
\end{array}\right) \quad h_{2}=\left(\begin{array}{ll}
0 & 0 \\
0 & 1
\end{array}\right) .
$$

We define two involutions as follows:

Definition 1. $X^{\prime}$ is the operator obtained from $X$ by the change $e \leftrightarrow f$ and perm $\circ X^{\circ}$ perm, where perm is the permutation switching the first and third factors.

Note that $(X Y)^{\prime}=Y^{\prime} X^{\prime}$.

Definition 2. $\bar{X}$ is the operator obtained from $X$ by the interchange $h_{1} \leftrightarrow h_{2}$ and $q \leftrightarrow q^{-1}$.

Note that $\overline{X Y}=\bar{Y} \bar{X}$.

One may easily check that $R^{\prime}=R$ (where the permutation acts on the first and third factors) and $\bar{R}=R$. Now define

$$
\begin{array}{ll}
A=R_{123} R_{124}, & B=R_{134} R_{234}, \\
C=R_{234} R_{134}, & D=R_{124} R_{123} .
\end{array}
$$

Proposition 3. We have the identities
a) $A=\bar{D}$,
b) $A=B^{\prime}$,
c) $C=D^{\prime}$,
d) $C=\bar{B}$.

Proof. (a) Decompose $R^{(3)}$ from the left and the right according to

$$
\begin{aligned}
R^{(3)} & =R^{(2)} \otimes h_{1}+R^{(2)-} \otimes h_{2}+\left(q-q^{-1}\right) R^{(2) e} \otimes f \\
& =h_{1} \otimes R^{(2)}+h_{2} \otimes R^{(2)-}+\left(q-q^{-1}\right) e \otimes R^{(2) f},
\end{aligned}
$$

where

$$
\begin{aligned}
& R^{(2) e}=q^{-1 / 2} h_{1} \otimes e-q^{1 / 2} h_{2} \otimes e-e \otimes h q^{-3 h / 2} \\
& R^{(2) f}=q^{-1 / 2} f \otimes h_{1}-q^{1 / 2} f \otimes h_{2}-h q^{-3 h / 2} \otimes f
\end{aligned}
$$


and $R^{(2)-}$ is $R^{(2)}$ with $q$ replaced by $q^{-1}$ (and coincides with the inverse of $R^{(2)}$ ). We will refer to the first and second decompositions in (3.3) as the right and the left decompositions respectively. Writing out $A=R_{123} R_{124}$ and $D=R_{124} R_{123}$ with respect to the right decomposition we see that we must establish four equations like

$$
\overline{R^{(2) e} R^{(2)}}=-R^{(2)-} R^{(2) e},
$$

but these in turn follow from $\overline{X Y}=\bar{Y} \bar{X}$ and $\overline{R^{(2) e}}=-R^{(2) e}$.

(b) The proof is entirely analogous to (a) but we now use $(X Y)^{\prime}=Y^{\prime} X^{\prime}$. Similarly, one may establish (c), (d).

The tetrahedral equation is the equation $A B=C D$. To verify it, we first decompose $A, B, C, D$ on both the left and the right to define:

$$
\begin{aligned}
A \equiv & h_{1} \otimes A_{11} \otimes h_{1}+h_{1} \otimes A_{12} \otimes h_{2}+h_{2} \otimes A_{21} \otimes h_{1}+h_{2} \otimes A_{22} \otimes h_{2} \\
& +h_{1} \otimes A_{1 f} \otimes f+h_{2} \otimes A_{2 f} \otimes f+e \otimes A_{e 1} \otimes h_{1}+e \otimes A_{e 2} \otimes h_{2}+e \otimes A_{e f} \otimes f,
\end{aligned}
$$

and similarly for $B, C, D$. The tetrahedral equation is now equivalent to three sets of equations as follows:

type 1:

$$
\begin{array}{ll}
A_{11} B_{11}=C_{11} D_{11}, & A_{12} B_{12}=C_{12} D_{12} \\
A_{22} B_{22}=C_{22} D_{22}, & A_{21} B_{21}=C_{21} D_{21}
\end{array}
$$

type 2:

$$
\begin{aligned}
& A_{12} B_{1 f}+A_{1 f} B_{11}=C_{12} D_{1 f}+C_{1 f} D_{11}, \\
& A_{22} B_{2 f}+A_{2 f} B_{21}=C_{22} D_{2 f}+C_{2 f} D_{21}, \\
& A_{11} B_{e 1}+A_{e 1} B_{21}=C_{11} D_{e 1}+C_{e 1} D_{21}, \\
& A_{12} B_{e 2}+A_{e 2} B_{22}=C_{12} D_{e 2}+C_{e 2} D_{22} .
\end{aligned}
$$

type 3:

$$
A_{1 f} B_{e 1}+A_{e 2} B_{2 f}+A_{e f} B_{21}+A_{12} B_{e f}=C_{1 f} D_{e 1}+C_{e 2} D_{2 f}+C_{e f} D_{21}+C_{12} D_{e f} .
$$

Under the $\mathbb{Z}_{2} \times \mathbb{Z}_{2}$ involutions the two lines in identities of type 1 are interchanged and all four identities of type 2 are interchanged, so it suffices to verify only one of these. Verification of the three types of identities proceeds by finding explicit formulae for $A_{11}$ etc. The decomposition of $A, B, C, D$ is most effectively carried out as follows. Write, for example,

$$
R_{123} R_{124}=h_{1} \otimes R_{12}^{(2)} R_{13}^{(2)}+h_{2} \otimes R_{12}^{(2)-} R_{13}^{(2)-}+\left(q-q^{-1}\right) e \otimes\left(R_{12}^{(2)} R_{13}^{(2) f}+R_{12}^{(2) f} R_{13}^{(2)-}\right) \text {. }
$$

Interestingly, we see that the first two terms are related to the two comultiplications $\Delta, \Delta^{\prime}[4]$ of the quantum group associated with (1.2). Exploiting this relation and computing the third term we obtain

$$
\begin{aligned}
R_{12}^{(2)} R_{13}^{(2)}= & (1 \otimes \Delta) R^{(2)}=h_{1} \otimes\left(q^{h / 2} \otimes q^{h / 2}\right)+h_{2} \otimes\left(q^{-h / 2} \otimes q^{-h / 2}\right) \\
& +q^{-1 / 2}\left(q-q^{-1}\right) e \otimes\left(q^{h / 2} \otimes f+f \otimes q^{-h / 2}\right), \\
R_{12}^{(2)-} R_{13}^{(2)-}= & \left(1 \otimes \Delta^{\prime}\right) R^{(2)-}=h_{1} \otimes\left(q^{-h / 2} \otimes q^{-h / 2}\right)+h_{2} \otimes\left(q^{h / 2} \otimes q^{h / 2}\right) \\
& -q^{1 / 2}\left(q-q^{-1}\right) e \otimes\left(q^{-h / 2} \otimes f+f \otimes q^{h / 2}\right),
\end{aligned}
$$




$$
\begin{aligned}
R_{12}^{(2)} R_{13}^{(2) f}+R_{12}^{(2) f} R_{13}^{(2)-}= & \left(q-q^{-1}\right)\left[\left(2 q^{-1} f \otimes h_{1}+\left(1-2 q^{-2}\right) h_{1} \otimes f+q^{2} h_{2} \otimes f\right) \otimes h_{1}\right. \\
& +\left(-2 q f \otimes h_{2}+q h_{2} \otimes f-q h_{1} \otimes f\right) \otimes h_{2} \\
& +\left(-q^{-1} h_{1} \otimes h_{1}-q^{-2} h_{1} \otimes h_{2}+\left(2 q^{2}-1\right) h_{2} \otimes h_{2}\right. \\
& \left.\left.+q^{-1} h_{2} \otimes h_{1}+\left(q^{2}-q^{-2}\right) e \otimes f\right) \otimes f\right] .
\end{aligned}
$$

From these equations one may find $A_{i j}$ and then by using Proposition $3, B_{i j}, C_{i j}, D_{i j}$. Finally, one may explicitly verify the relevant identities in (3.4-3.6), thus completing the proof.

Several important issues are raised by Theorem 2 . One may wonder if the quantum solution is, in some sense, unique. In fact, it is possible to write down a nontrivial two-parameter solution for which the solution of Theorem 2 is a special case. Moreover, there is the problem of extending our solution to other representations, and the related question of the existence of a universal $R$ matrix in the tetrahedral case. If such a thing exists one could wonder if there is an explicit formula in terms of generators of the universal enveloping algebra of $s l(2)$ or of $s l(2)_{q}$. More generally, our solution of (3.1) should have some important underlying algebraic meaning - which we believe begins to make its appearance in the proof of Theorem 2 - but which has yet to be elucidated ${ }^{3}$.

\section{An Obstruction for $n=4$}

We now consider the quantization of the nondegenerate classical solutions for $n=4$, i.e., we will try to quantize the classical solution

$$
\begin{aligned}
r= & h \otimes h \otimes h \otimes h+4 h \otimes e \otimes f+4 h \otimes e \otimes f \otimes h \\
& +4 e \otimes f \otimes h \otimes h-4 h \otimes e \otimes h \otimes f-4 e \otimes h \otimes f \otimes h \\
& +4 e \otimes h \otimes h \otimes f+16 e \otimes f \otimes e \otimes f
\end{aligned}
$$

in the tensor product of 2-dimensional representations of $\operatorname{sl}(2)$. The distinction of $n=4$ from the smaller dimensions is reflected in

Theorem 3. The classical $r$ matrix (4.1) in the two-dimensional representation of $\operatorname{sl}(2)$ cannot be quantized.

Proof. We will show that no quantum $R$ matrix exists by considering its perturbation expansion:

$$
R=1+t r+t^{2} r^{(2)}+t^{3} r^{(3)}+\cdots,
$$

where $r$ is given by (4.1) in the two-dimensional representation. The quantum Yang-Baxter equation is equivalent to an infinite set of equations obtained from the various powers of $t$, in particular we will be interested in the equations at order $t^{3}$ and $t^{4}$. At order $t^{3}$ we must solve the equation

$$
\sum_{i<j}\left(\left[r_{i}, r_{j}^{(2)}\right]+\left[r_{i}^{(2)}, r_{j}\right]\right)+\sum_{i<j<k}\left(r_{i} r_{j} r_{k}-r_{k} r_{j} r_{i}\right)=0 .
$$

${ }^{3}$ One of the more obvious possibilities is to define an algebra with cubic relations via $R_{123} L_{12} L_{13} L_{23}=L_{23} L_{13} L_{12} R_{123}$ in a fashion entirely analogous to [6]. One soon finds that other relations on the $L$ 's are necessary 
Using the identity

$$
a b c-c b a=\frac{1}{2}(\{a,[b, c]\}+\{b,[a, c]\}+\{c,[a, b]\})
$$

and the classical Yang-Baxter equation for $r$ one verifies that $r^{(2)}=\frac{1}{2} r^{2}$ is a solution of (4.3). Thus, to find an obstruction one must consider the order four equation, and this in turn requires a knowledge of the most general solution of (4.3). Hence we need to know the space of solutions $p$ of

$$
\sum_{i<j}\left(\left[r_{i}, p_{j}\right]+\left[p_{i}, r_{j}\right]\right)=0
$$

From the results of Sect. 2 it is clear that there is at least a four-dimensional space of solutions. Since (4.1) is just $S^{4}(A=1+\sqrt{-1} k)$ we may always take $p=w 1+x r+$ $y S^{4}(i+\sqrt{-1} j)+z S^{4}(i-\sqrt{-1} j)$ for any $w, x, y, z$. Direct examination of the equation (which is an upper triangular $32 \times 32$ matrix of equations) using symbolic manipulation on a computer reveals that the true solution space is in fact five-dimensional, the most general homogeneous solution being

$$
p=w 1+x r+y S^{4}(i+\sqrt{-1} j)+z S^{4}(i-\sqrt{-1} j)+u e \otimes e \otimes f \otimes f,
$$

so that the most general possibility for $r^{(2)}$ is

$$
r^{(2)}=\frac{1}{2} r^{2}+p
$$

We now turn to the order 4 equation:

$$
\begin{aligned}
& \sum_{i<j}\left(\left[r_{i}, r_{j}^{(3)}\right]+\left[r_{i}^{(3)}, r_{j}\right]\right)+\sum_{i<j<k<l}\left(r_{i} r_{j} r_{k} r_{l}-r_{l} r_{k} r_{j} r_{i}\right) \\
& \quad+\sum_{i<j<k}\left(\left(r_{i} r_{j} r_{k}^{(2)}-r_{k}^{(2)} r_{j} r_{i}\right)+\left(r_{i} r_{j}^{(2)} r_{k}-r_{k} r_{j}^{(2)} r_{i}\right)+\left(r_{i}^{(2)} r_{j} r_{k}-r_{k} r_{j} r_{i}^{(2)}\right)\right) \\
& \quad+\sum_{i<j}\left[r_{i}^{(2)}, r_{j}^{(2)}\right]=0,
\end{aligned}
$$

where we must substitute (4.5). This equation may be simplified by several considerations. First, taking into account the possibility of redefining $R^{(4)}$ by an overall scale, and of redefining the variable $t$ we see that, without loss of generality, we may put $w=x=0$. Furthermore, from the choice of solution (4.1) we see that the equations and variables admit a $\mathbb{Z}_{2}$ grading with $1, k$ considered even and $i, j$ considered odd. In particular, it suffices to prove that there is no solution for the even part of $r^{(3)}$. Since the variables $y, z$ are odd, and since $S^{4}(i \pm \sqrt{-1} j)$ are solutions of the classical 4-simplex equation we see that these odd terms in (4.5) only contribute to the last term in (4.6). One may now try to solve the linear equations (4.6), with the parameters $u, y z$ kept arbitrary, using symbolic manipulation on a computer. One rapidly finds that the equations are inconsistent, establishing our result.

The above result, and its proof, raises three rather evident questions. First, there is the (ugly) logical possibility that there are other representations besides spin $1 / 2$ which can be quantized. We believe this is impossible but have not ruled it our. Second, our method of proof used a computer and was decidedly heavy-handed. A better understanding of the reason for the obstruction is certainly called for. Third, for reasons discussed at length in the introduction, it is natural 
to conjecture that the classical solutions cannot be quantized in any representation for $n>4$ as well as for $n=4$.

\section{Further Questions}

We believe that the present paper is only a very first insight into a new kind of mathematical structure. Our results raise many new questions. In addition to those discussed above we would like to mention the following:

1. Do the degenerate solutions of the classical triangular equation (e.g. taking $a=0$ in (2.1)) admit interesting quantizations?

2. What are the solutions to the classical triangular equation for other Lie algebras?

3. Is there a quantization for classical solutions for other Lie algebras?

4. Is the solution of Theorem 2 a special case of a more general solution of the tetrahedral equations involving a spectral parameter? If so, what integrable 3-dimensional statistical mechanical model is it associated with?

5. Is the solution of Theorem 2 related to the elliptic solutions of the Yang-Baxter equation?

6. Is the solution of Theorem 2 related to gauge theories in four dimensions?

\section{Appendix A. Relation to Zamolodchikov's Equation}

The equation studied in this paper is similar to, but not equivalent to Zamolodchikov's equation for the "scattering of straight strings" [7]. In this appendix we explain the difference.

In the scattering of straight strings the basic scattering amplitude is the $S$-matrix for a three-string collision which may be interpreted as an operator $R \in \operatorname{End}\left(V_{1} \otimes V_{2} \otimes V_{3}\right)$ where $V_{i}$ are the state spaces of strings $1,2,3$. The intersections of straight strings divide each string into a number of segments, and Zamolodchikov assigns a string state by "coloring" each segment, where the colors may be taken as basis vectors in some vector space $W$. In contrast to the case of particles in $1+1$ dimensions, the state space $V$ available to any given string depends on the number of other strings in its universe so that, e.g., in the presence of $n$ strings each string has a state space $V=W^{\otimes n}$. Thus, the basic 3 string scattering amplitude is an element

$$
R \in \operatorname{End}\left(\left(W^{\otimes 3}\right) \otimes\left(W^{\otimes 3}\right) \otimes\left(W^{\otimes 3}\right)\right) .
$$

(Moreover, Zamolodchikov assumes that $R$ is diagonal on the first and third factor of each space $W^{\otimes 3}$.) Then, to describe 3-string scattering in a universe containing 4 strings we must choose an embedding.

$$
\operatorname{End}\left(\left(W^{\otimes 3}\right) \otimes\left(W^{\otimes 3}\right) \otimes\left(W^{\otimes 3}\right)\right) \rightarrow \operatorname{End}\left(\left(W^{\otimes 4}\right) \otimes\left(W^{\otimes 4}\right) \otimes\left(W^{\otimes 4}\right)\right)
$$

to define a 3-string scattering operator

$$
R \in \operatorname{End}\left(\left(W^{\otimes 4}\right) \otimes\left(W^{\otimes 4}\right) \otimes\left(W^{\otimes 4}\right)\right) .
$$

The choice of embedding is restricted by the requirement that the scattering be local. We choose the embedding which is the identity on the first copy of $W$ in 
each of the spaces $W^{\otimes 4}$. The tetrahedron equation may now be formulated in terms of the consistency condition for four string scattering as a sequence of consecutive 3-string scatterings. Since the different 3-string scattering processes in the equation require different embeddings

$$
\operatorname{End}\left(\left(W^{\otimes 3}\right) \otimes\left(W^{\otimes 3}\right) \otimes\left(W^{\otimes 3}\right)\right) \rightarrow \operatorname{End}\left(\left(W^{\otimes 4}\right) \otimes\left(W^{\otimes 4}\right) \otimes\left(W^{\otimes 4}\right)\right)
$$

we must also introduce the permutation operator $P \in \operatorname{End}\left(W^{\otimes 4}\right)$ defined by

$$
P: w_{1} \otimes w_{2} \otimes w_{3} \otimes w_{4} \rightarrow w_{4} \otimes w_{1} \otimes w_{2} \otimes w_{3}
$$

where $w_{i} \in W$. We can now finally write the tetrahedron equations as:

$$
\begin{aligned}
& \left(P_{2} P_{3} P_{4} R_{234} P_{2}^{-1} P_{3}^{-1} P_{4}^{-1}\right)\left(P_{1} R_{134} P_{1}^{-1}\right)\left(P_{4} R_{124} P_{4}^{-1}\right)\left(P_{1} P_{2} P_{3} R_{123} P_{1}^{-1} P_{2}^{-1} P_{3}^{-1}\right) \\
& \quad=R_{123}\left(P_{1} P_{2} R_{124} P_{1}^{-1} P_{2}^{-1}\right)\left(P_{3} P_{4} R_{134} P_{3}^{-1} P_{4}^{-1}\right) R_{234},
\end{aligned}
$$

where $P_{1}=P \otimes 1 \otimes 1 \otimes 1$ etc. In addition, the consistency of the scattering of straight strings requires further equations like $R_{234} R_{135}=R_{135} R_{234}$ in the case of five-string scattering. This is satisfied by Zamolodchikov's three-string amplitude essentially because $R$ is local and is diagonal on certain factor spaces, as indicated above. Unfortunately the solution of Theorem 2 does not satisfy this locality requirement. Hence, to apply our results to statistical mechanics and geometry will require some further ideas. Nevertheless, the basic strategy of this paper can be used to investigate Zamolodchikov's equations. The reason is that, if we consider the classical limit of (A.4) for tensor powers of any representation, the extra permutations in (A.4) are irrelevant. Thus our results on the classical equations carry over unchanged.

Acknowledgements. We would like to thank K. Rabe for much help. We also thank J. Fisher, H. Garland, N. Read, N. Seiberg, R. Shankar, V. Turaev, and O. Viro for discussions. The work of I.F. was supported by NSF grant DMS-8906772 and by a Guggenheim Foundation Fellowship. The work of G.M. was supported by DOE grant DE-AC02-76ER03075 and by a Presidential Young Investigator Award.

\section{References}

1. Baxter, R. J.: Exactly solved models in statistical mechanics. New York: Academic Press 1982

2. Witten, E.: Quantum field theory and the Jones polynomial. Commun. Math. Phys. 121, 351 (1989)

3. Turaev, V., Reshetikhin, N. Yu.: Invariants of 3-manifolds via link polynomials and quantum groups, preprint

4. Drinfeld, V. G.: Quantum groups. Proceedings of the International Congress of Mathematicians 1986, p. 789

5. Jimbo, M.: Introduction to the Yang-Baxter Equation. Int. J. Mod. Phys. A4, 3759 (1989)

6. Faddeev, L. D., Reshetikhin, N. Yu., Takhtadjan, L. A.: Quantization of Lie groups and Lie algebras. Leningrad Math. J. 1, 193 (1990)

7. Zamolodchikov, A. B.: Tetrahedra equations and integrable systems in three-dimensional space. Sov. Phys. JETP 52, 325 (1980); Commun. Math. Phys. 79, 489 (1981)

8. Bazhanov, V. V., Stroganov, Yu. G.: Theor. Math. Phys. 52, 685 (1982); Nucl. Phys. B205, 505 (1982)

9. Manin, Yu. I., Schechtman, V. V.: Higher Bruhat orders, related to the symmetric group. Funct. Anal. Appl. 20, 148 (1986); Arrangements of hyperplanes, higher braid groups and higher Bruhat orders. Adv. Studies Pure Math. 17, 289 (1989) 
10. Baxter, R. J.: On Zamolodchikov's solution of the tetrahedron equations. Commun. Math. Phys. 88, 185 (1983); Partition function of the three-dimensional Zamolodchikov model. Phys. Rev. Lett. 53, 1795 (1984); The Yang-Baxter equations and the Zamolodchikov model. Physica 2D, 321 (1986); Baxter, R. J., Quispel, G. R. W.: Hamiltonian limit of the 3D Zamolodchikov model. J. Stat. Phys. 58, 411 (1990)

11. Maillet, J. M., Nijhoff, F.: The tetrahedron equation and the four-simplex equation. Phys. Lett. A134, 221 (1989); Integrability for multidimensional lattice models. preprint CERNTH-5332/89

12. We thank $\mathrm{O}$. Viro, $\mathrm{V}$. Turaev for conservations on this point

13. Atiyah, M. F.: New invariants of 3- and 4-dimensional manifolds. Proc. Symp. Pure Math. 48, 285 (1988)

14. Witten, E.: The search for higher symmetry in string theory. IASSNS-HEP-88/55, Lecture at the meeting on string theory of the Royal Society, London 1988

15. Witten, E.: Geometry and quantum field theory IASSNS-HEP-89/17, Lecture at the Centennial Meeting of the American Mathematical Society, Providence, August 1988

Communicated by N. Yu. Reshetikhin 
